Esko Ruokonen

Ilkka Parviainen

Stephan M. Jakob

Silvia Nunes

Maija Kaukonen

Stephen T. Shepherd

Toni Sarapohja

J. Raymond Bratty

Jukka Takala

For the "Dexmedetomidine

for Continuous Sedation"

Investigators

\title{
Dexmedetomidine versus propofol/midazolam for long-term sedation during mechanical ventilation
}

\section{S. Nunes}

Department of Intensive Care,

Tampere University Hospital,

P.O. Box 2000, 33521 Tampere, Finland

Published online: 16 September 2008

(C) Springer-Verlag 2008

Registered at ClinicalTrials.gov, number NCT00226785.

A complete list of investigators and study centers appears in the Appendix.

This study was sponsored by Orion Pharma, Helsinki, Finland, through research contracts negotiated directly with the participating hospitals.

Electronic supplementary material The online version of this article (doi:10.1007/s00134-008-1296-0) contains supplementary material, which is available to authorized users.

\section{E. Ruokonen · I. Parviainen}

Department of Anesthesiology

and Intensive Care Medicine,

Kuopio University Hospital,

P.O. Box 1777, 70211 Kuopio, Finland

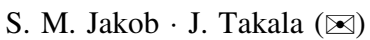

Department of Intensive Care Medicine, Bern University Hospital and University of Bern, 3010 Bern, Switzerland e-mail: jukka.takala@insel.ch

Tel.: +41-31-6324144

Fax: +41-31-6324100

\section{Kaukonen}

Units of Intensive Care Medicine, Division of Anesthesiology and Intensive Care Medicine, Department of Surgery, Helsinki University Central Hospital, P.O. Box 340, 00029 HUS, Helsinki, Finland

S. T. Shepherd - T. Sarapohja · J. R. Bratty Orion Pharma, P.O. Box 65,

02101 Espoo, Finland

\begin{abstract}
Purpose: To compare dexmedetomidine (DEX) with standard care (SC, either propofol or midazolam) for long-term sedation in terms of maintaining target sedation and length of intensive care unit (ICU) stay. Methods: A pilot, phase III, double-blind multicenter study in randomized medical and surgical patients $(n=85)$ within the first $72 \mathrm{~h}$ of ICU stay with an expected ICU stay of $\geq 48 \mathrm{~h}$ and sedation need for $\geq 24 \mathrm{~h}$ after randomization. Patients were assigned to either DEX $\left(\leq 1.4 \mu \mathrm{g} \mathrm{kg}^{-1} \mathrm{~h}^{-1} ; n=41\right)$ or SC $(n=44)$, with daily sedation stops.
\end{abstract}

Results: Non-inferiority of DEX versus SC was not confirmed. Target Richmond agitation-sedation score (RASS) was reached a median of $64 \%$ (DEX) and 63\% (SC) of the sedation time (ns). The length of ICU stay was similar in DEX and SC. Patients with RASS target 0-3 (DEX $78 \%$, SC 80\%) were at target sedation $74 \%$ (DEX) and 64\% (SC) of the time (ns), whereas those with RASS target -4 or less reached the target $42 \%$ (DEX) and $62 \%$ (SC) of the time $(P=.006)$. Post hoc analyses suggested shorter duration of mechanical ventilation for $\operatorname{DEX}(P=0.025)$. Conclusions: This pilot study suggests that in long-term sedation, DEX is comparable to $\mathrm{SC}$ in maintaining sedation targets of RASS 0 to -3 but not suitable for deep sedation (RASS -4 or less). DEX had no effect on length of ICU stay. Its effects on other relevant clinical outcomes, such as duration of mechanical ventilation, should be tested further.

Keywords Dexmedetomidine . Sedatives - Deep sedation . Conscious sedation - Intensive care . Critical care $\cdot$ Mechanical ventilation

\section{Introduction}

Sedation is used in the intensive care unit (ICU) to enhance patient comfort and safety, facilitate mechanical ventilation and reduce oxygen demands. Side effects may include prolonged mechanical ventilation and ICU stay [1-3]. Although sedation protocols and daily sedation stops may reduce excessive sedation due to drug 
accumulation and shorten ICU stay [1, 2], the management of long-term sedation with traditional sedatives remains problematic.

Dexmedetomidine is a sedative with high affinity for $\alpha_{2}$-adrenoreceptors [4]. It sedates via interaction with the locus ceruleus, and has less effect on arousability and patient interaction $[5,6]$. In post-surgical patients, dexmedetomidine does not interfere with respiration rate, or arterial oxygenation and carbon dioxide pressure [7]. The cardiovascular effects of dexmedetomidine are dose dependent, and include decreases in heart rate, cardiac output, and arterial and pulmonary arterial pressures $[8,9]$.

Dexmedetomidine has been studied in the ICU mainly for sedation after surgery up to $24 \mathrm{~h}[6,10,11]$. Data from small uncontrolled studies suggest that dexmedetomidine may be safe and effective for long-term sedation as well $[12,13]$. In a small uncontrolled series, dexmedetomidine infused continuously for up to 7 days was an effective sedative and analgesic-sparing drug [12]. To obtain sufficient sedation for several days, dexmedetomidine doses had to be increased up to three times the initially scheduled doses [13]. Recently, the first randomized, controlled trial on the use of dexmedetomidine for up to 5 days found that as compared to lorazepam infusion, sedation by dexmedetomidine was associated with increased days alive without delirium or coma in mechanically ventilated ICU patients [14].

The hierarchical co-primary objectives of this study were, first, to assess if sedation with dexmedetomidine (higher doses and/or longer duration than approved) is as good as standard sedation (with propofol/midazolam and daily sedation stops) in maintaining target sedation level in long-stay ICU patients, and second, to compare the effects of dexmedetomidine and standard sedation on length of ICU stay.

\section{Materials and methods}

For details, see electronic supplementary material (ESM). This multicenter, prospective, randomized, double-blind, double-dummy, active comparator study was approved by the Finnish national research ethics committee and by the Ethics Committee of the Canton of Bern, Switzerland. Written informed consent was obtained either from the patient's family ( 3 centers) or from the family and an independent physician (1 center). An independent data safety monitoring board had full access to the data. The study treatment was started within $72 \mathrm{~h}$ of ICU admission. Patients were randomized to either continue their current standard care (midazolam or propofol; SC) or to switch to dexmedetomidine (DEX). Randomization was stratified for study center, current sedative, sedation target (Richmond Agitation-Sedation Scale [15]; RASS)
0 to -3 vs. RASS -4 , and admission type (medical vs. postoperative/trauma). Study treatments were titrated to individual sedation targets. Pain was treated with fentanyl boluses, as clinically indicated. Rescue medication (first-line rescue propofol for patients receiving midazolam, midazolam for those receiving propofol before randomization; further rescue medication decided by clinician in charge) could be given if needed to achieve the target depth of sedation. Need for sedation was assessed at a daily sedation stop (used routinely in all centers before the study), conducted at the same time each day. The first sedation stop was $12-36 \mathrm{~h}$ after randomization, depending on the time of randomization. The study treatment lasted a maximum of 14 days from randomization and the follow-up for 45 days (Fig. 1).

The main inclusion criteria were age $\geq 18$ years, mechanical ventilation, need for sedation for $\geq 24 \mathrm{~h}$ after randomization and an expected ICU stay $\geq 48 \mathrm{~h}$. The exclusion criteria were acute severe neurological disorder, mean arterial pressure $<55 \mathrm{mmHg}$ despite volume and vasopressors, heart rate $<50$ beats min $^{-1}$; AV-conduction block II-III (unless pacemaker installed), hepatic Sequential Organ Failure Assessment (SOFA) score [16] $>2$, bilirubin $>101 \mu \mathrm{mol} \mathrm{L}{ }^{-1}$, lactation or positive pregnancy test, muscle relaxation, loss of hearing or vision, any other condition interfering with RASS assessment, use of $\alpha_{2}$-agonists or antagonists at the time of randomization.

Dexmedetomidine and dexmedetomidine dummy $(0.9 \% \mathrm{NaCl})$, propofol $2 \%$ and propofol dummy $(0.9 \%$ $\mathrm{NaCl})$ and midazolam $(0.1 \%)$ and midazolam dummy $(0.9 \% \mathrm{NaCl})$ were prepared by personnel not involved in the study or the patient's care; infusion systems for propofol and its dummy were nontransparent. In order to maintain the target RASS, dexmedetomidine was infused without a loading dose at $0.8 \mu \mathrm{g} \mathrm{kg}^{-1} \mathrm{~h}^{-1}$ for $1 \mathrm{~h}$ and then adjusted stepwise at $0.25,0.5,0.8,1.1$, and $1.4 \mu \mathrm{g} \mathrm{kg}^{-1} \mathrm{~h}^{-1}$; propofol was infused at $2.4 \mathrm{mg} \mathrm{kg}^{-1} \mathrm{~h}^{-1}$ for $1 \mathrm{~h}$ and then adjusted stepwise at $0.8,1.6,2.4,3.2$, and $4.0 \mathrm{mg} \mathrm{kg}^{-1} \mathrm{~h}^{-1}$. Depending on standard care at time of randomization, midazolam was given either as intravenous boluses (1-2 mg), starting at 3 boluses per hour for $1 \mathrm{~h}$, and thereafter $1-4$ boluses per hour, and if not sufficient as continuous infusion of $0.2 \mathrm{mg} \mathrm{kg}^{-1} \mathrm{~h}^{-1}$, or as a continuous infusion at $0.12 \mathrm{mg} \mathrm{kg}^{-1} \mathrm{~h}^{-1}$ for $1 \mathrm{~h}$, followed by adjustments at 0.04, 0.08, 0.12, 0.16, and $0.20 \mathrm{mg} \mathrm{kg}^{-1} \mathrm{~h}^{-1}$. The initial dose could be reduced, if considered necessary by the treating clinician.

Primary efficacy variables Depth of sedation was assessed using the RASS (routinely used in all centers before study), and the target RASS was determined before starting study treatment and at the daily sedation stops. RASS was assessed every $2 \mathrm{~h}$ during the treatment. Length of ICU stay was measured from ICU admission and from randomization to medically fit for discharge. 
Fig. 1 Schematic diagram of study protocol
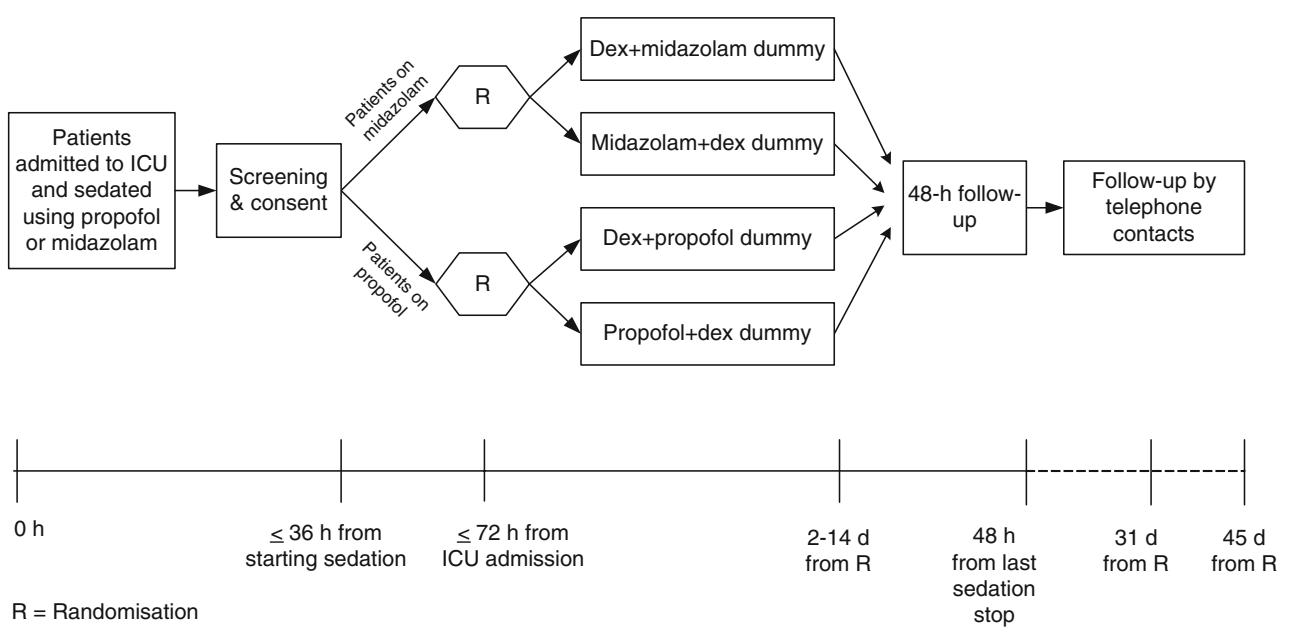

Dex $=$ Dexmedetomidine

Double blind-double dummy design

- expected length of stay $\geq 48$ hours and need for sedation $\geq 24$ hours from randomisation

- drugs titrated to target sedation range using Richmond Agitation-Sedation Scale (RASS)

- need for continued sedation assessed at daily sedation stop

- primary comparison: "usual care" (combined midazolam + propofol groups) vs. combined dex groups

- hospital discharge recorded
Secondary efficacy variables included nurses' assessment of arousal, cooperation, and ability to communicate pain with visual analogue scales (VAS), duration of mechanical ventilation, weaning time, ventilator-free days in the ICU, length of hospital stay (actual and until medically fit for discharge), functional recovery during hospitalization [17], and need for rescue medication. For safety variables and adverse events (AEs), see ESM. Delirium was assessed daily by trained study personnel [18].

\section{Statistical methods}

The hierarchical co-primary objectives were, first, to evaluate the non-inferiority of DEX versus SC in maintaining target RASS, and second, to compare effect of DEX versus SC on length of ICU stay. Maintenance of target sedation (proportion of sedation time within RASS target, without rescue medication) was analyzed using analysis of co-variance (ANCOVA; main effects treatment, stratification factors used in randomization; covariate time from admission to randomization). Noninferiority of DEX versus SC was defined (using perprotocol data set to avoid bias towards non-inferiority, and one-sided $97.5 \%$ confidence interval; CI) as $<10 \%$ difference between the treatments (lower CI of the estimated $\mathrm{DEX} / \mathrm{SC}>0.90$ ).

Intention-to-treat data were used for all other efficacy variables. The length of ICU stay to medically fit for discharge was analyzed with Kaplan-Meier method and Cox's proportional-hazards regression model (including stratification factors and time from admission to randomization). Duration of mechanical ventilation and time to hospital discharge were analyzed using Cox's proportional-hazards regression model and ventilator-free days in ICU using generalized linear model (effect for treatment and length of ICU stay as offset variables) with post hoc adjustments for baseline stratification factors, and the VAS results using ANCOVA (effect for treatment and baseline as covariate).

Imputation rules for length of ICU stay and duration of mechanical ventilation were modified post hoc to include all scenarios for missing data (see ESM). The results in the main text are given using the modified imputation rules.

The plan was to recruit 90 patients as a separate pilot study and to continue recruiting up to 900 patients (450 each in DEX and SC). After 85 pilot patients, the study was discontinued and analyzed for subsequent design of two pivotal studies.

Categorical data were analyzed using Fisher's exact test or the Chi square test. SAS statistics software was used (SAS version 9.1, SAS Institute Inc., Cary, NC, USA).

\section{Results}

Ninety-five patients were screened and 85 were randomized (intention-to-treat patients) to receive dexmedetomidine (DEX; $n=41$ ) or to continue their previous standard care (SC; $n=44)$ (Table 1; Fig. 2). Seventynine patients $(93 \%$; 38 DEX; $41 \mathrm{SC})$ were included in the per-protocol analysis (Fig. 2). The occurrence rate and 
Table 1 Demographics, the presence of infection at ICU admission and occurrence rate and severity of organ failures at baseline

\begin{tabular}{|c|c|c|c|c|}
\hline \multicolumn{2}{|l|}{ Variable } & Dexmedetomidine $N=41$ & Midazolam/propofol $N=44$ & $P$ value $^{\mathrm{a}}$ \\
\hline \multicolumn{2}{|l|}{ Age (years) } & Median 64 , range $18-83$ & Median 68 , range $18-83$ & \\
\hline \multicolumn{2}{|l|}{ Female/male (n) } & $9 / 32$ & $6 / 38$ & \\
\hline \multicolumn{2}{|l|}{ Medical patients } & $21(51 \%)$ & $24(55 \%)$ & \\
\hline \multicolumn{2}{|l|}{ Infection at ICU admission } & $23(56 \%)$ & $20(45 \%)$ & 0.388 \\
\hline \multirow[t]{2}{*}{ Respiratory system } & SOFA >1 & $41(100 \%)$ & $44(100 \%)$ & 1.000 \\
\hline & SOFA 3 or 4 & $36(87.8 \%)$ & $38(86.4 \%)$ & \\
\hline \multirow[t]{2}{*}{ Coagulation } & SOFA $>1$ & $15(36.6 \%)$ & $15(34.1 \%)$ & 0.174 \\
\hline & SOFA 0 or 1 & $26(63.4 \%)$ & $29(65.9 \%)$ & \\
\hline \multirow[t]{2}{*}{ Liver } & SOFA $>1$ & $5(12.2 \%)$ & $3(6.8 \%)$ & 0.206 \\
\hline & SOFA 0 or 1 & $36(87.8 \%)$ & $41(93.2 \%)$ & \\
\hline \multirow[t]{2}{*}{ Cardiovascular system } & SOFA $>1$ & $28(68.3 \%)$ & $31(70.5 \%)$ & 0.182 \\
\hline & SOFA 0 or 1 & $13(31.7 \%)$ & $13(29.5 \%)$ & \\
\hline \multirow[t]{2}{*}{ Central nervous system ${ }^{\mathrm{b}}$} & SOFA $>1$ & $2(4.9 \%)$ & $2(4.5 \%)$ & 0.384 \\
\hline & SOFA 0 or 1 & $38(92.7 \%)$ & $40(90.9 \%)$ & \\
\hline \multirow[t]{2}{*}{ Kidney } & SOFA $>1$ & $13(31.7 \%)$ & $12(27.3 \%)$ & 0.170 \\
\hline & SOFA 0 or 1 & $28(68.3 \%)$ & $32(72.7 \%)$ & \\
\hline
\end{tabular}

A sequential organ failure assessment score (SOFA) $>1$ was considered as organ failure. Respiratory system SOFA 3 and 4 indicate $\mathrm{PaO}_{2} / \mathrm{FiO}_{2}<200$ and $<100$, respectively, during mechanical ventilation a Fisher's exact test

b Missing central nervous system SOFA scores at baseline, dexmedetomidine $n=1$, midazolam/propofol $n=2$
Fig. 2 Randomization, completion of study treatment, reasons for discontinuation of study treatment, and patient outcomes in intention-to-treat population; inclusions into perprotocol population and reasons for exclusions. Note: one patient in each study arm was lost to follow-up after day 31 ; both were still hospitalized after discharge to another hospital, and were considered as being in hospital at 45-day follow-up

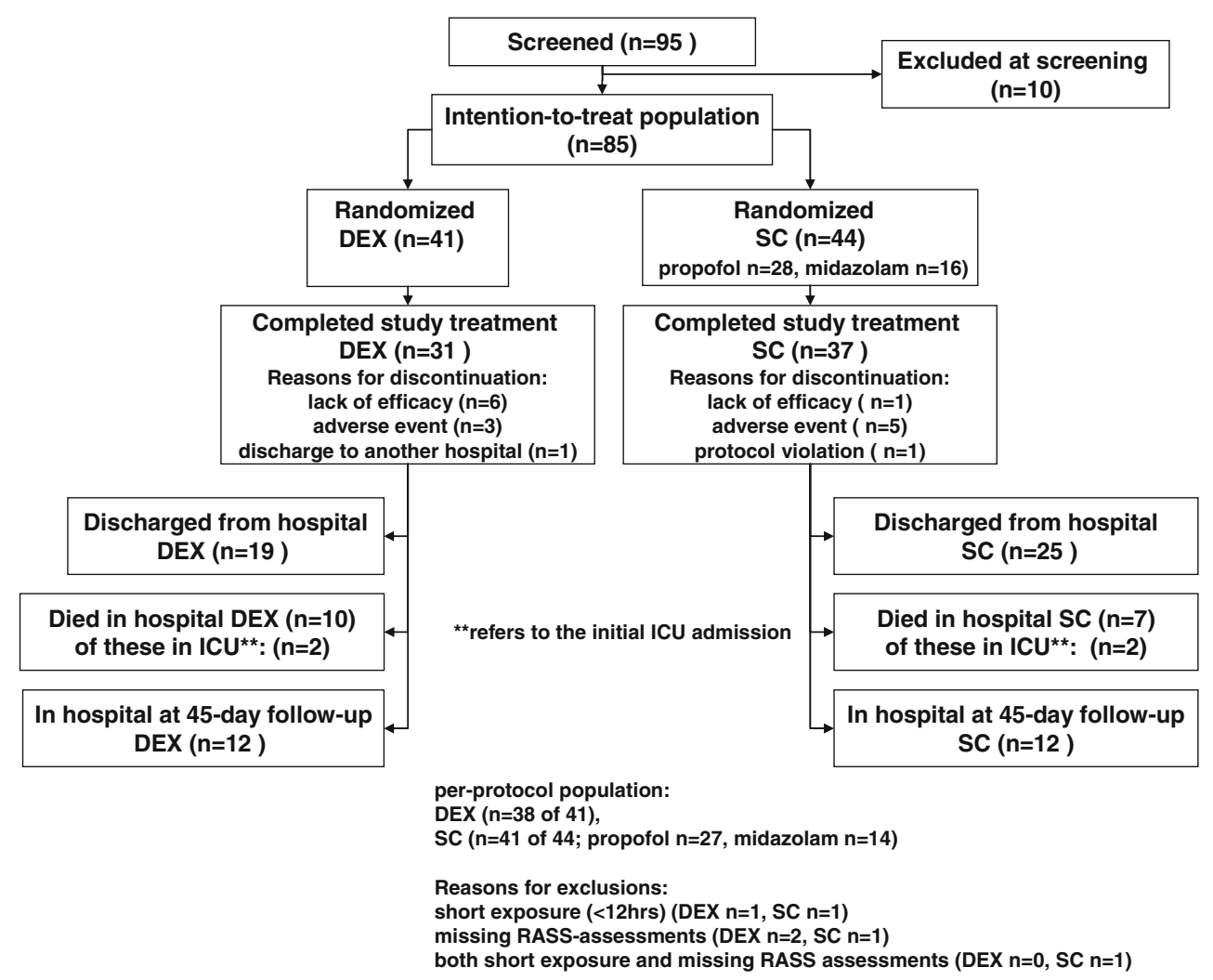

Non-inferiority of DEX versus SC was not confirmed. DEX and SC resulted in similar proportions of time at target sedation without rescue medication (DEX 64 vs. SC 63\%; Table 3). The estimated ratio between DEX/SC in time at target sedation was 0.97 (95\% CI 0.79-1.15). The lower limit of the $95 \%$ CI did not reach the limit of short exposure ( $<12 \mathrm{hrs}$ ) (DEX $n=1, S C n=1$ )
missing RASS-assessments (DEX $n=2, S C n=1$ )

both short exposure and missing RASS assessments (DEX $n=0, S C n=1$ ) severity of organ failures at baseline were comparable between the treatment groups (Table 1). Duration and dose of study drugs is given in Table 2. Use of fentanyl was similar in DEX and SC (Table 2). There was no significant difference between treatments in need for rescue medication. 
Table 2 Duration (excluding sedation stops) and dose of study drugs, use of fentanyl, and duration of sedation stops; median (range)

\begin{tabular}{|c|c|c|}
\hline & Dexmedetomidine & Standard care \\
\hline $\begin{array}{l}\text { Duration of study drug } \\
\text { administration (h) }\end{array}$ & $40(3-198)$ & $\begin{array}{l}\text { Midazolam boluses } \\
24 \text { (8-83) } \\
\text { midazolam infusion } \\
27 \text { (4-105) } \\
\text { propofol infusion } \\
61(15-256)\end{array}$ \\
\hline $\begin{array}{l}\text { Dose of study drug } \\
\qquad\left(\mu \mathrm{gg}^{-1} \mathrm{~h}^{-1}\right)\end{array}$ & $0.8(0.3-1.4)$ & $\begin{array}{l}\text { Midazolam boluses } \\
37 \text { (27-76) } \\
\text { midazolam infusion } \\
87 \text { (44-160) } \\
\text { propofol infusion } \\
2067(845-3659)\end{array}$ \\
\hline $\begin{array}{l}\text { Cumulative dose } \\
\text { of fentanyl (mg) }\end{array}$ & $1.5(0.1-20)$ & $1.6(0.1-9)$ \\
\hline $\begin{array}{l}\text { Duration of sedation } \\
\text { stop (h) }\end{array}$ & $2.3(0-29.0)$ & $\begin{array}{l}1.1(0-49.1) \\
\text { midazolam } 10.3(0-49.1) \\
\text { propofol } 0.7(0-28.1)\end{array}$ \\
\hline
\end{tabular}

non-inferiority of DEX versus SC (at least 0.90). The lengths of stay from admission and randomization to medically fit for discharge from the ICU were similar in both treatment groups (Table 3; Fig. 3).

Target sedation at baseline influenced the time spent at RASS without rescue medication $(P=0.057$ for treatment $\times$ target RASS interaction). Of those with target RASS -4 , DEX patients $(n=8)$ spent $42 \%$ (median; range $4-64 \%$ ) of the time at target RASS, and SC patients $(n=8) 62 \%(0-85)$. DEX patients with baseline target RASS of 0 to $-3(n=30)$ were at target RASS $74 \%$ of the time (median; range 0-99\%) and SC patients $(n=33) 64 \%$ (9-100\%).

Sedation stops (Table 2) were performed on $71 \%$ of days in the DEX group and $70 \%$ in the SC group. The most common reason a sedation stop was not performed was clinical contraindication, e.g., severe gas exchange disturbance or high risk of hemodynamic instability (DEX $21.4 \%$, SC $20.9 \%$ of sedation days). The most common reasons to restart sedation after a sedation stop were poor tolerance of the endotracheal tube or mechanical ventilation (DEX 18 events, SC 30 events), agitation or anxiety (DEX 19 events, SC 31 events), and cardiovascular instability (DEX 6 events, SC 11 events).

The median duration of mechanical ventilation was $77.2 \mathrm{~h}(17.5-338.8 \mathrm{~h})$ in the DEX group and $110.6 \mathrm{~h}$ $(20.1-675.0 \mathrm{~h})$ in the SC group $(P=0.109$; Fig. 3$)$. When adjusted post hoc for study center, sedative agent before randomization, and target sedation level, the duration of mechanical ventilation was shorter with DEX $(P=0.025)$. In patients with light to moderate sedation (RASS 0 to -3 ), the median duration of mechanical ventilation in the DEX group was $70.2 \mathrm{~h}(17.5-225.4 \mathrm{~h})$ versus median $93.7 \mathrm{~h}(20.1-675.0 \mathrm{~h})$ in the SC group $(P=0.135$, after post hoc adjustment $P=0.027)$. There were no significant differences between the groups in duration of weaning, number of ventilator-free days, or time to discharge from the hospital (for details, see ESM). Ten DEX patients $(24.4 \%)$ and 7 SC patients $(15.9 \%)$ died between randomization and day 45 follow-up.

The VAS score of nurses' assessment of patient communication (lower scores indicate better response)

Table 3 Percentage of time at target sedation without rescue medication; median (range) and length of ICU stay to medically fit for discharge; days, median (range)

\begin{tabular}{|c|c|c|c|}
\hline $\begin{array}{l}\text { Dexmedetomidine } \\
(N=38 / 41)\end{array}$ & $\begin{array}{l}\text { Standard care } \\
\text { (midazolam or propofol) } \\
(N=41 / 44)\end{array}$ & $P$ value $^{\mathrm{a}}$ & $\begin{array}{l}\text { Hazards } \\
\text { ratio }^{\mathrm{a}}\end{array}$ \\
\hline
\end{tabular}

\begin{tabular}{|c|c|c|c|c|c|}
\hline Time at target sedation $(\%)$ & $64(0-99)$ & $\begin{array}{l}63(0-100) \\
\text { Midazolam } 63(0-100) \\
\text { Propofol } 63(9-100)\end{array}$ & & & \\
\hline Estimated ratio DEX/SC ${ }^{\mathrm{b}}$ & & 0.97 & & & $0.79-1.15$ \\
\hline \multicolumn{6}{|c|}{ Length of ICU stay to medically fit for discharge (days) } \\
\hline From admission & $6.6(2.2-20.7)$ & $\begin{array}{l}6.8(2.6-30.8) \\
\text { Midazolam } 6.9(3.3-30.8) \\
\text { Propofol } 6.8(2.6-28.2)\end{array}$ & 0.275 & 0.766 & $0.476-1.235$ \\
\hline From randomization & $5.5(1.7-19.5)$ & $\begin{array}{l}5.7(1.7-29.0) \\
\text { Midazolam } 5.8(1.8-29.0) \\
\text { Propofol 5.2 (1.7-27.1) }\end{array}$ & 0.411 & 0.821 & $0.512-1.315$ \\
\hline For medical patients & $5.0(1.7-19.5)$ & $\begin{array}{l}4.9(1.8-29.0) \\
\text { Midazolam } 6.0(1.8-29.0) \\
\text { Propofol } 4.7(1.8-27.1)\end{array}$ & 0.016 & 0.427 & $0.213-0.854$ \\
\hline For postoperative and/or trauma patients & $5.7(2.0-16.7)$ & $\begin{array}{l}5.9(1.7-16.8) \\
\text { Midazolam } 5.8(2.9-16.8) \\
\text { Propofol } 6.7(1.7-11.5)\end{array}$ & 0.062 & 2.091 & $0.964-4.533$ \\
\hline
\end{tabular}




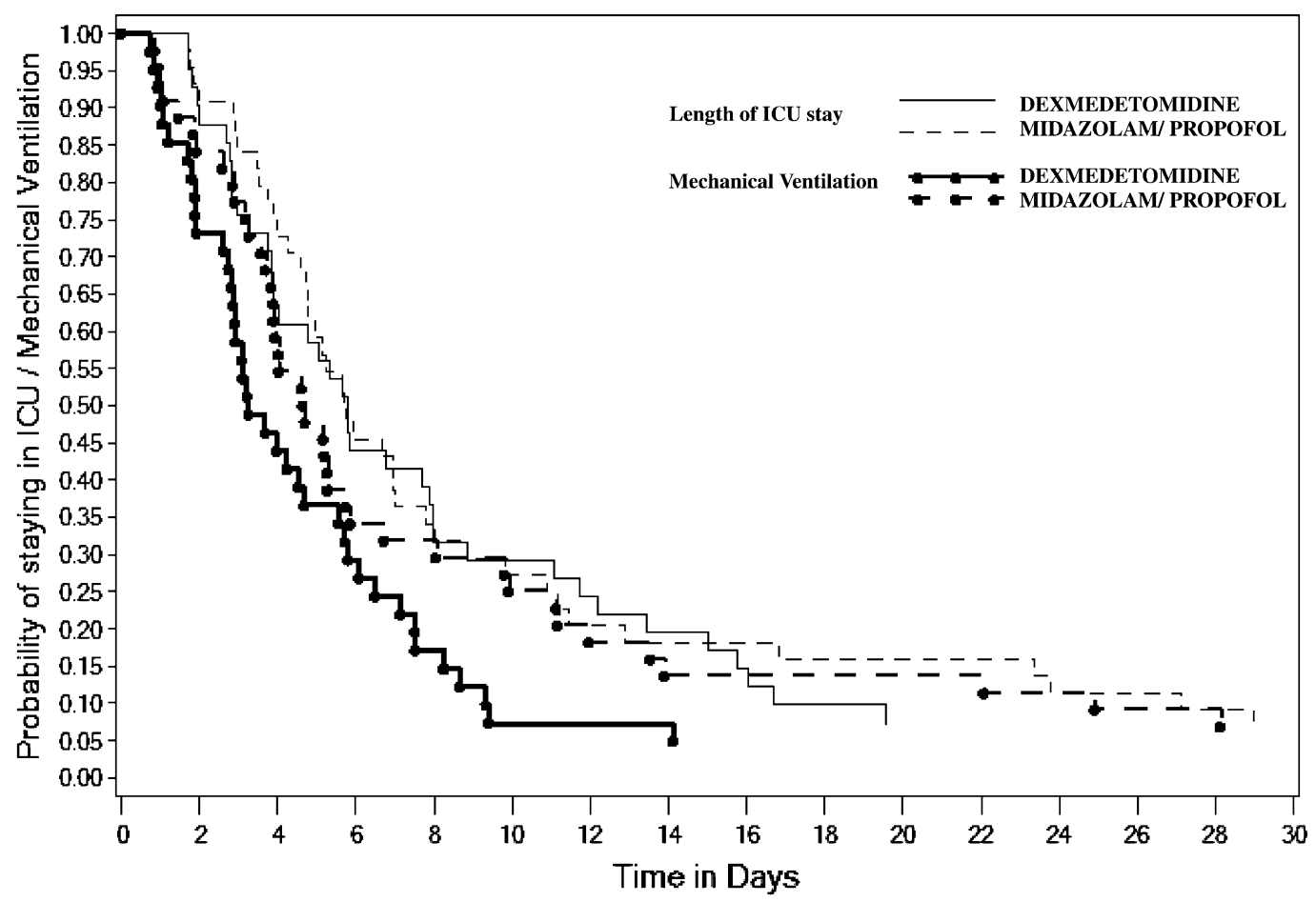

Patients at risk $(\mathrm{n})$

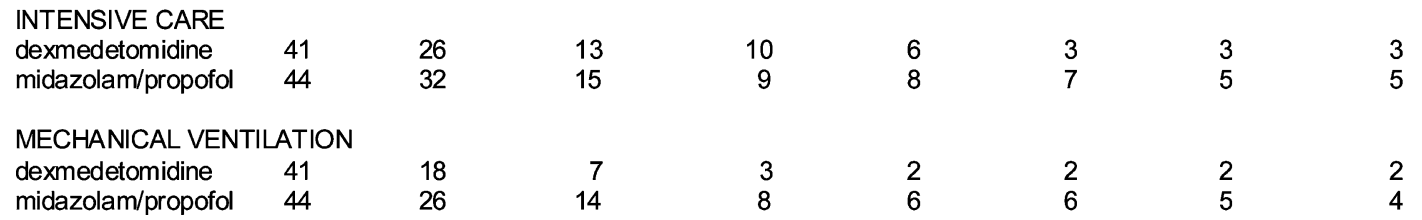

Fig. 3 Probability of remaining on mechanical ventilation and staying in the intensive care unit (Kaplan-Meier curves)

demonstrated that DEX patients were more arousable, more cooperative, and better able to communicate their pain than SC patients [mean (SD) sum of VAS scores DEX $30.6(21.0)$ vs. SC 47.5 (27.7); $P<0.001$ for the sum and each VAS].

Delirium was more common in the DEX group (43.9 vs. $25.0 \%$ in SC; $P=0.035$ ) when analyzed as the combined endpoint of CAM-ICU and adverse events (AEs) of delirium and confusion. However, more CAMICU assessments were performed in the DEX group (106 DEX vs. 84 SC assessments), and the proportion of positive CAM-ICU results was comparable (DEX 17.0 vs. SC $17.9 \%$, n.s.). During the follow-up to ICU discharge, no significant difference was observed in the occurrence rate of positive RASS scores (DEX 26 vs. SC 32\%).

Serious adverse events (SAE) were equally common: 41 DEX patients (including 3 patients with bradycardia, 1 cardiac arrest 5 days after end of treatment) and 47 SC patients had SAEs. There were no differences between groups in the incidence of any SAE (see ESM). All DEX patients and $95.5 \%$ of SC patients had AEs (a total of 296 in DEX and 275 in SC). At day 2, a cardiovascular
SOFA score $>1$ was more common in DEX (77.5 vs. $56.1 \%$ in SC; $P=0.024$ ), indicating more frequent use of vasopressors/inotropes in the first $24 \mathrm{~h}$ of study treatment.

\section{Discussion}

This randomized, controlled, double-blind, doubledummy trial of dexmedetomidine for long-term sedation extended the duration of dexmedetomidine use and compared its efficacy in maintaining target sedation levels with standard care in a broad spectrum of long-stay intensive care patients. The standard sedation was optimized by using daily sedation stops and frequent sedation scoring. As a pilot study, this study was not powered for its co-primary outcomes (non-inferiority in maintaining target sedation levels and length of ICU stay). Based on its results, two pivotal studies have been designed and are currently running (http://www.ClinicalTrials.gov, identifiers NCT00481312, NCT00479661). Despite its pilot 
character, this study provides relevant information on the safety, efficacy, and characteristics of dexmedetomidine in long-term sedation. The only previous randomized controlled trial on dexmedetomidine for long-term sedation (up to 5 days) in ICU patients used lorazepam infusion without sedation stops as comparator [14]. In contrast, patients in our study continued their previous care, including sedation stops, as comparator.

Although the non-inferiority of dexmedetomidine versus standard care was not confirmed, the proportion of time at target sedation without rescue medication and the need for rescue medication were similar in the treatment groups. Dexmedetomidine was more applicable for moderate sedation than deep sedation despite the use of a higher maximum dose of dexmedetomidine $\left(1.4 \mu \mathrm{g} \mathrm{kg}^{-1} \mathrm{~h}^{-1}\right)$ than in most previous studies [10-12]. A need for additional sedatives has been reported with dexmedetomidine at doses of up to $2.5 \mu \mathrm{g} \mathrm{kg}^{-1} \mathrm{~h}^{-1}$ [13]. Dexmedetomidine was associated with less coma (defined as RASS -4 to -5 ) and markedly increased fentanyl needs in patients with RASS target -3 or deeper [14]. When viewed together with its short-term use $[6,10,11]$ and any long-term use [12-14], our results suggest that dexmedetomidine should be further assessed for mild to moderate sedation rather than as sole sedative for deep sedation.

Use of sedation protocols with daily sedation stops and sedation scores has been able to reduce the duration of mechanical ventilation and intensive care $[1,2]$. Pandharipande [14] observed a tendency toward more ventilator-free days with dexmedetomidine. Since no sedation stops were used, the more frequent oversedation with lorazepam may have contributed. In the present study, sedation stops and score-targeted sedation were used in both treatment groups, and $2 / 3$ of patients in SC received propofol, a short-acting agent. Still, the duration of mechanical ventilation tended to be shorter in patients receiving dexmedetomidine. Since the reduction of duration of mechanical ventilation in the present study was significant only after post hoc baseline stratification adjustments, this finding must be regarded as hypothesisgenerating. Furthermore, shorter mechanical ventilation did not translate to reduced duration of ICU stay. The relevance of this finding remains speculative; conceivably, shorter mechanical ventilation may, e.g., reduce nosocomial infections and need for tracheostomy. The medication costs of sedation with DEX are likely to be higher than those of SC. These must be balanced with the potential impact on other components of intensive care and hospitalization. The overall costs of sedation with dexmedetomidine need to be evaluated.

The similar length of ICU stay despite the tendency toward shorter mechanical ventilation in the DEX group deserves consideration. Delirium, agitation, or anxiety could prolong the length of ICU stay in already extubated patients. This is unlikely, since the occurrence rate of agitation (positive RASS) was similar in the groups after stopping mechanical ventilation.

Delirium, confusional state, and positive CAM-ICU (but not the proportion of positive CAM-ICU of those assessed) were more frequent in DEX, whereas sedation needed to be restarted after a sedation stop due to agitation or anxiety more frequently in SC. A retrospective analysis suggested increased pain and agitation when dexmedetomidine was begun to reduce standard sedation [19]. On the other hand, dexmedetomidine has been proposed to reduce agitation [20] and delirium [21], and alphareceptor agonists have been used to treat delirium [22].

Pandharipande [14] used a composite primary endpoint of delirium and coma-free days to compare dexmedetomidine with lorazepam infusion in 106 ventilated patients. Dexmedetomidine resulted in more days alive without delirium and coma, and more time with actual RASS score within one point of target. Since coma but not delirium was reduced, it is conceivable that lorazepam infusion resulting in inadvertently deep sedation may have contributed. Since RASS was assessed twice daily without formal sedation stops, and the comparator was long-acting [14], their results in achieving target sedation cannot be directly compared with ours. Despite differences in study design, the findings of these two double-blind, randomized, controlled trials support the concept that dexmedetomidine can provide adequate long-term sedation. At least in moderate sedation, dexmedetomidine may reduce oversedation as compared to either benzodiazepines or propofol (standard care).

Difficulties in communication and pain assessment may contribute to oversedation and suboptimal analgesia. Similar to our results in healthy subjects [5], DEX patients were more arousable, more cooperative, and could better communicate pain than patients receiving standard care. Whether this improves patients' perception of sedation is controversial. Both positive perceptions [6] and increased discomfort and sleeping difficulties as compared to propofol at similar patient-rated awareness [11] have been reported.

Dexmedetomidine was well tolerated hemodynamically. Nevertheless, increased cardiovascular SOFA score was more common during the first day in DEX. Dexmedetomidine resulted in concentration-dependent decreases in heart rate, cardiac output, and cerebral blood flow in healthy subjects $[8,9,23]$ and in lower heart rate but similar blood pressure compared to propofol after cardiac surgery [11]. We observed no signs of cardiovascular instability and/or rebound after cessation of sedation.

As a pilot study, the sample size is a main limitation. The standard care comparator included the option of either propofol or midazolam instead of one comparator. Accordingly, the standard care consisted of two 
subgroups receiving pharmacologically very different agents. Although this is a limitation, such standard care group represents a broad range of patients and clinical practice, and may provide a better estimate of the potential effects of dexmedetomidine in clinical routine. A further potential limitation is that opioids were administered on clinical grounds, and despite similar patterns in DEX and SC, differences in sedation may have been masked, a problem common with all sedation trials.

In conclusion, dexmedetomidine appears safe and comparable to current sedation practice for long-term sedation, but not suitable as the sole agent for deep sedation (RASS -4 or less). Dexmedetomidine enhances the patient's ability to communicate. Large randomized clinical trials to test its effects on relevant clinical outcomes, such as mechanical ventilation, are underway.

\section{Appendix}

List of investigators and study centers

\section{Study Chair}

Jukka Takala, MD, PhD

Department of Intensive Care Medicine, Inselspital, Bern University Hospital, and University of Bern, Bern, Switzerland

\section{Principal investigators}

Esko Ruokonen, MD, PhD

Department of Anesthesiology and Intensive Care Medicine, Kuopio University Hospital, Kuopio, Finland

Stephan M. Jakob, MD, PhD

Department of Intensive Care Medicine, Inselspital, Bern University Hospital, and University of Bern, Bern, Switzerland

\section{Participating centers and investigators \\ Bern}

Investigators

Anna Brunello, MD

Barbara Haeberli

Research nurses

Klaus Maier

Jürgen Rohner

Judith Kaufmann

Department of Intensive Care Medicine, Inselspital, Bern University Hospital, and University of Bern, Bern, Switzerland

\section{Helsinki}

Investigator

Kirsi-Maija Kaukonen, MD, PhD
Research Nurse

Maiju Salovaara-Hiltunen, RN

Units of Intensive Care Medicine, Division of Anesthesiology and Intensive Care Medicine, Department of Surgery, Helsinki University Central Hospital, Helsinki, Finland

\section{Kuopio}

Investigators

Esko Ruokonen, $\mathrm{MD}, \mathrm{PhD}$

Ilkka Parviainen, $\mathrm{MD}, \mathrm{PhD}$

Ari Uusaro, MD, PhD

Kirsimarja Metsävainio, MD

Stepani Bendel, MD

Jouni Kurola, MD, PhD

Valdo Toome, MD

Research nurses

Sari Rahikainen, RN

Elina Halonen, RN

Heikki Ahonen, RN

Seija Laitinen

Department of Anesthesiology and Intensive Care

Medicine, Kuopio University Hospital

\section{Tampere}

Investigators

Silvia Nunes, MD, PhD

Sari Karlsson, MD

Pertti Arvola, MD, PhD

Markku Suvela, MD

Jyrki Tenhunen, MD, PhD

Riitta Halmela, MD

Research nurses

Anna-Liina Korkala, RN

Minna-Liisa Peltola, RN

Samuli Kortelainen, RN

Department of Intensive Care, Tampere University Hospital

\section{Investigators from study sponsor Orion Pharma}

Stephen T. Shepherd, MA, PhD, DipClinSci (study director)

J Raymond Bratty, BSc, MB BCh, FFPM (Clinical Program Leader)

Pasi Pohjanjousi, MSc (Biostatistician)

Toni Sarapohja, MSc (Biostatistics and Data Management Program Leader)

Tuula Ahtola-Sätilä, MSc (Pharm), DDS (Clinical Pharmacokineticist)

Marja Kelloniemi, Diploma in Systems Design (Data Manager)

Angela Ruck, BSc, PhD, MICR (Lead Monitor) 


\section{References}

1. Brook AD, Ahrens TS, Schaiff R, Prentice D, Sherman G, Shannon W, Kollef MH (1999) Effect of a nursingimplemented sedation protocol on the duration of mechanical ventilation. Crit Care Med 27:2609-2615

2. Kress JP, Pohlman AS, O'Connor MF, Hall JB (2000) Daily interruption of sedative infusions in critically ill patients undergoing mechanical ventilation. N Engl J Med 342:1471-1477

3. Sydow M, Neumann P (1999) Sedation for the critically ill. Intensive Care Med 25:634-636

4. Virtanen R, Savola JM, Saano V, Nyman L (1988) Characterization of the selectivity, specificity and potency of medetomidine as an alpha 2adrenoceptor agonist. Eur J Pharmacol 150:9-14

5. Hall JE, Uhrich TD, Barney JA, Arain SR, Ebert TJ (2000) Sedative, amnestic, and analgesic properties of small-dose dexmedetomidine infusions. Anesth Analg 90:699-705

6. Venn RM, Grounds RM (2001) Comparison between dexmedetomidine and propofol for sedation in the intensive care unit: patient and clinician perceptions. Br J Anaesth 87:684-690

7. Venn RM, Hell J, Grounds RM (2000) Respiratory effects of dexmedetomidine in the surgical patient requiring intensive care. Crit Care 4:302-308

8. Bloor BC, Ward DS, Belleville JP, Maze M (1992) Effects of intravenous dexmedetomidine in humans. II. Hemodynamic changes. Anesthesiology 77:1134-1142

9. Ebert TJ, Hall JE, Barney JA, Uhrich TD, Colinco MD (2000) The effects of increasing plasma concentrations of dexmedetomidine in humans. Anesthesiology 93:382-394

10. Herr DL, Sum-Ping ST, England M (2003) ICU sedation after coronary artery bypass graft surgery: dexmedetomidine-based versus propofol-based sedation regimens. J Cardiothorac Vasc Anesth 17:576-584
11. Corbett SM, Rebuck JA, Greene CM, Callas PW, Neale BW, Healey MA, Leavitt BJ (2005) Dexmedetomidine does not improve patient satisfaction when compared with propofol during mechanical ventilation. Crit Care Med 33:940-945

12. Shehabi Y, Ruettimann U, Adamson H, Innes R, Ickeringill M (2004) Dexmedetomidine infusion for more than 24 hours in critically ill patients: sedative and cardiovascular effects. Intensive Care Med 30:2188-2196

13. Venn M, Newman J, Grounds M (2003) A phase II study to evaluate the efficacy of dexmedetomidine for sedation in the medical intensive care unit. Intensive Care Med 29:201-207

14. Pandharipande PP, Pun BT, Herr DL, Maze M, Girard TD, Miller RR, Shintani AK, Thompson JL, Jackson JC, Deppen SA, Stiles RA, Dittus RS, Bernard GR, Ely EW (2007) Effect of sedation with dexmedetomidine vs. lorazepam on acute brain dysfunction in mechanically ventilated patients: the MENDS randomized controlled trial. JAMA 298:2644-2653

15. Sessler CN, Gosnell MS, Grap MJ, Brophy GM, O'Neal PV, Keane KA, Tesoro EP, Elswick RK (2002) The Richmond Agitation-Sedation Scale: validity and reliability in adult intensive care unit patients. Am J Respir Crit Care Med 166:1338-1344

16. Vincent JL, Moreno R, Takala J, Willatts S, De Mendonça A, Bruining H, Reinhart CK, Suter PM, Thijs LG (1996) The SOFA (Sepsis-related Organ Failure Assessment) score to describe organ dysfunction/failure. Intensive Care Med 22:707-710

17. Bennett-Guerrero E, Welsby I, Dunn TJ, Young LR, Wahl TA, Diers TL, Phillips-Bute BG, Newman MF, Mythen MG (1999) The use of a postoperative morbidity survey to evaluate patients with prolonged hospitalization after routine, moderaterisk, elective surgery. Anesth Analg 89:514-519
18. Ely EW, Inouye SK, Bernard GR, Gordon S, Francis J, May L, Truman B, Speroff T, Gautam S, Margolin R, Hart RP, Dittus R (2001) Delirium in mechanically ventilated patients: validity and reliability of the confusion assessment method for the intensive care unit (CAM-ICU. JAMA 286:2703-2710

19. MacLaren R, Forrest LK, Kiser TH (2007) Adjunctive dexmedetomidine therapy in the intensive care unit: a retrospective assessment of impact on sedative and analgesic requirements, levels of sedation and analgesia, and ventilatory and hemodynamic parameters. Pharmacotherapy 27:351-359

20. Siobal MS, Kallet RH, Kivett VA, Tang JF (2006) Use of dexmedetomidine to facilitate extubation in surgical intensive-care-unit patients who failed previous weaning attempts following prolonged mechanical ventilation: a pilot study. Respir Care 51:492-496

21. Pandharipande P, Ely EW (2005) Narcotic-based sedation regimens for critically ill mechanically ventilated patients. Crit Care 9:247-248

22. Mayo-Smith MF (1997)

Pharmacological management of alcohol withdrawal. A meta-analysis and evidence-based practice guideline. American Society of Addiction Medicine Working Group on Pharmacological Management of Alcohol Withdrawal. JAMA 278:144-151

23. Prielipp RC, Wall MH, Tobin JR, Groban L, Cannon MA, Fahey FH, Gage HD, Stump DA, James RL, Bennett J, Butterworth J (2002) Dexmedetomidine-induced sedation in volunteers decreases regional and global cerebral blood flow. Anesth Analg 95:1052-1059 